
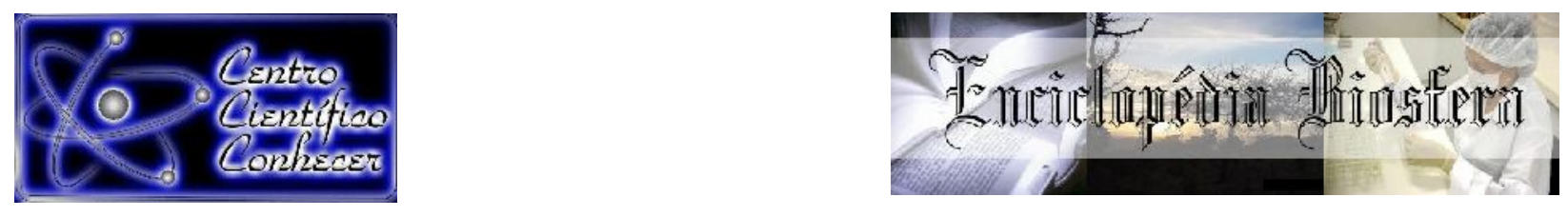

\title{
O EFEITO DA CARGA ELETROSTÁTICA NA FILTRAÇÃO DE PARTíCULAS DE CIMENTO EM FILTROS DE MANGAS
}

Flávia Matias Oliveira da Silva ${ }^{1}$, Marcos Vinicius Rodrigues ${ }^{2}$ e Mônica Lopes Aguiar ${ }^{3}$

${ }^{1}$ Mestra em Engenharia Química, Universidade Federal de São Carlos, Brasil. (flaviamoliveira04@gmail.com)

${ }^{2}$ Doutor em Eng. Química - Professor na Universidade Federal de Alfenas, Brasil,

${ }^{3}$ Doutora em Eng. Química - Professora Titular na Universidade Federal de São Carlos, Brasil.

Recebido em: 06/04/2019 - Aprovado em: 10/06/2019 - Publicado em: 30/06/2019 DOI: 10.18677/EnciBio_2019A185

\begin{abstract}
RESUMO
$\mathrm{Na}$ busca por uma maior eficiência e menor gasto energético na operação de filtração, a junção de dois equipamentos de separação gás-sólido torna-se uma alternativa viável para melhorar o desempenho destes equipamentos. O presente trabalho teve como objetivo verificar a influência da carga eletrostática nas partículas durante a formação da torta de filtração de gases. Como material particulado foi utilizado cimento com diâmetro volumétrico de 14 e $20 \mu \mathrm{m}$ e dois níveis de carregamento eletrostático: $-1,0$ e $-2,0 \mathrm{kV}$. Através dos ensaios realizados foi possível observar que, com a aplicação da carga eletrostática, ocorreu uma diminuição na resistência específica da torta de filtração. Pode-se dizer que o tamanho da partícula afeta diretamente a eficiência da filtração, e que a carga, pode aumentar a eficiência de coleta das partículas e diminuir a perda de carga durante a filtração de gases.
\end{abstract}

PALAVRAS - CHAVE: Carga eletrostática; Cimento; Filtração; Filtros de Mangas; Material particulado.

\section{THE ELECTROSTATIC EFFECT IN FILTRATION OF CEMENT PARTICLES IN BAG FILTERS}

\begin{abstract}
In the search for greater filtration efficiency and lower filtration operating energy expenditure, the junction of two or more gas-solid separation equipment becomes a viable alternative to improve the operational performance of the filters. In this study, it was proposed to investigate the influence of the level of electrostatic charge on the particle information of the dust cake. Cement was used as a particulate material with a volumetric diameter of 14 and $20 \mu \mathrm{m}$ and two levels of the voltage of -1 and $-2 \mathrm{kV}$. Possible shows changed during the filtration. It can be said that the particle size directly affects the filtration efficiency, and the charger it can increase the efficiency the collect to particulate and decrease pressure drop during filtration of gases.
\end{abstract}

KEYWORDS: Bag Filter, Cement, Corona Charger, Filtration, Particulate matter. 


\section{INTRODUÇÃO}

Inovando a eficiência na filtração de gases e particulados, novas fibras foram desenvolvidas para a coleta de partículas, alcançando alta eficiência até mesmo para nano partículas. $O$ interesse por tecnologias alternativas para melhorar a eficiência dos equipamentos já existentes no mercado está crescendo e um método que está sendo explorado atualmente são os filtros eletrostáticos híbridos.

O filtro eletrostático híbrido consiste em um precipitador eletrostático seguido de um filtro de mangas, este equipamento tem um processo de filtração diferente da filtração convencional. Na primeira etapa, as partículas são somente carregadas com a descarga eletrostática enquanto passam pelo precipitador eletrostático, na segunda etapa estas partículas seguem para o filtro de mangas, que neste caso, é o único equipamento de filtração utilizado. Os dois equipamentos de filtração utilizados já são conhecidos pela elevada eficiência de coleta para materiais particulados, quando utilizados separadamente.

$O$ interesse por este tema foi devido aos estudos de alguns pesquisadores (OLIVEIRA et al. 2015; FENG et al. 2016; TU et al. 2016; JAWOREK et al., 2017; RODRIGUES et al., 2017; FENG et al., 2018; JAWOREK et al., 2019) que vêm demostrando que a presença de cargas eletrostáticas nos filtros de mangas pode aumentar o desempenho da filtração aumentando à eficiência de coleta do material particulado e diminuindo a perda de carga. Frederick (1974) e Givechi et al., (2015) investigaram a aplicação de cargas eletrostáticas durante a filtração gás-sólido, obtendo resultados em que a eficiência de coleta aumentou. No entanto, trabalhar com a geração de cargas eletrostáticas é extremamente difícil, principalmente no controle dessas cargas, por não se ter um comportamento estável na maioria das vezes.

Cargas artificiais ou a combinação do precipitador eletrostático e os filtros de tecidos tem recebido cada vez mais atenção, como um meio para melhorar a eficiência de coleta na filtração. Essa técnica tem maior influência para partículas ultrafinas, embora um efeito positivo fosse observado para filtração de partículas de $10 \mu \mathrm{m}$ (FREDERICK, 1974). Sabe-se que durante a filtração de gases a presença de material particulado gera carga eletrostática através da triboeletrificação, ou seja, o atrito entre as partículas, e entre a tubulação gera cargas naturais. Deste modo as partículas são eletrizadas naturalmente e este fator foi um incentivo para que estudos sobre a indução de cargas eletrostáticas "genéricas" fossem realizados (BROWN et al., 1988).

No trabalho de Frederick (1974), foram avaliados filtros de tecidos com e sem aplicação de um campo elétrico. $O$ autor utilizou um filtro de mangas (tecido de carbono), colocado dentro de uma gaiola de Faraday para conduzir a carga eletrostática com uma tensão de 7,5 kV os testes foram feitos com partículas de cimento. Observou-se que a aplicação da tensão provocou uma redução na resistência específica da torta e, aumentou a eficiência de coleta. Frederick concluiu que o filtro de mangas com aplicação de cargas artificiais pode aumentar a coleta do material particulado e reduzir em até $24 \%$ a resistência da torta de filtração. Este comportamento ocorreu porque ao introduzir cargas eletrostáticas nas partículas durante a filtração, à descarga corona feita pelo eletrodo fez com que as partículas se aglomerassem formando partículas maiores, que se rearranjaram de maneira diferente no meio filtrante, facilitando a sua retenção pelo filtro de tecido. Anos depois este mesmo comportamento foi observado por Oliveira et al., (2015).

Givehchi et al., (2015), aplicaram uma carga eletrostática em partículas de $\mathrm{NaCl}$ de $10 \mathrm{~nm}$ a $100 \mathrm{~nm}$, os resultados mostraram que a carga eletrostática 
desempenhou um grande papel na filtração de nano aerossol, que é diferente de teorias de filtração convencionais. A eficiência de filtração aumentou $30 \% \mathrm{com} o$ aumento do tamanho das nano partículas. Os resultados também mostraram uma correlação positiva entre a eficiência de separação devido às forças eletrostáticas e o tempo de permanência das partículas no fluxo de ar durante a filtração, ou seja, a velocidade de filtração também influenciou na eficiência do processo.

Feng et al., (2016), utilizando um sistema híbrido de filtração eletrostática (SHFE), que combina um sistema de precipitador eletrostático (SPE) e um filtro fibroso instalado a jusante do SPE, usando um carregador eletrostático com cargas aplicadas de $-6,5 ;-8,5$ e -10 kV, apontaram uma maior eficiência eletrostática no filtro fibroso decorrente das partículas de dioctilftalato carregadas que atravessavam - ESP. Esses autores utilizando um filtro de polietileno e partículas de 0,4 $\mu \mathrm{m}$, verificaram que a eficiência aumentou de $5 \%$ para $60 \%$ devido a maior tensão aplicada e ao mecanismo inercial.

Tu et al., (2016) exploraram o efeito das cargas eletrostáticas nas partículas de cinzas durante a filtração, utilizando dois tipos de filtros comerciais, filtros fibrosos e de membrana. Para o filtro fibroso, a carga nas partículas aumentou a eficiência de limpeza do filtro e mudou o ponto inicial de formação da torta de filtração, reduzindo assim a emissão de particulados. A porosidade média das tortas de filtração foram ambas aumentadas com o aumento das cargas nas partículas, diminuindo assim a queda de pressão.

Rodrigues et al., (2017) a fim de verificarem a deposição inicial das partículas, com diâmetro médio de Stokes de 3,35 m, em filtros de polipropileno, aplicaram cargas elétricas de -3 e $-6 \mathrm{kV}$ nas partículas de rocha fosfática durante a filtração de gases, com apenas um eletrodo de descarga, observaram que a eficiência da filtração aumentou e também houve uma redução nos valores de queda de pressão.

Jaworek et al., (2019) estudaram trabalhos relacionados ao sistema de filtração eletrostático híbrido no controle da emissão de particulados. Os autores fizeram um 'review' com todos os trabalhos relevantes ligados ao tema iniciando em 1930 até os dias atuais. O trabalho relata sistemas de operação dos diferentes filtros híbridos utilizados hoje em dia, descrevendo minuciosamente todas suas características desde o precipitador eletrostático até a torta de filtração formada nas fibras dos tecidos. O 'review' deixou claro que, os sistemas de filtração eletrostático híbrido parecem ser os dispositivos mais eficientes utilizados para a remoção de partículas de cinzas voláteis em centrais elétricas a carvão no futuro.

Embora estudos sobre a influência da carga eletrostática na filtração de gases estejam sendo realizados, a maioria desses estudos está focada na deposição das partículas no meio filtrante e na fase inicial da filtração, onde os mecanismos de coleta ainda são atuantes. No entanto, a influência da carga eletrostática na formação da torta de filtração de gases ainda precisa ser mais explorada, visto que, a utilização de dois equipamentos de filtração de gases em paralelo (filtro híbrido) é mais eficiente que a filtração convencional, trazendo muitos benefícios para 0 processo, como prolongar a vida útil das mangas, mantendo o sistema funcionando por um período maior, isto pode gerar um excelente ganho no custo benefício do processo.

Assim, este trabalho teve como objetivo avaliar o efeito da presença de cargas eletrostáticas na formação das tortas de filtração de gases. Para tal finalidade, foram avaliadas: a queda de pressão no filtro em função da massa de material retido, a porosidade teórica e experimental e a deposição das partículas na torta e no meio filtrante para partículas de cimento com diâmetro volumétrico de 14 e $20 \mu \mathrm{m}$ e dois 
níveis de carregamento eletrostático -1,- e -2 kV. Optou-se por estas tensões com valores abaixo do efeito corona para investigar a influência da carga eletrostática nas partículas com intuito de somente eletrizar as partículas, sem ocorrer a coleta de partículas no precipitador eletrostático.

\section{MATERIAIS E MÉTODOS}

Uma visão geral do sistema experimental utilizado nos ensaios de filtração é apresentada na Figura 1. O sistema consiste em um alimentador de pó tipo prato giratório com venturi, dispositivo de controle de umidade, precipitador eletrostático, suporte de filtro e um módulo de controle/aquisição de dados.

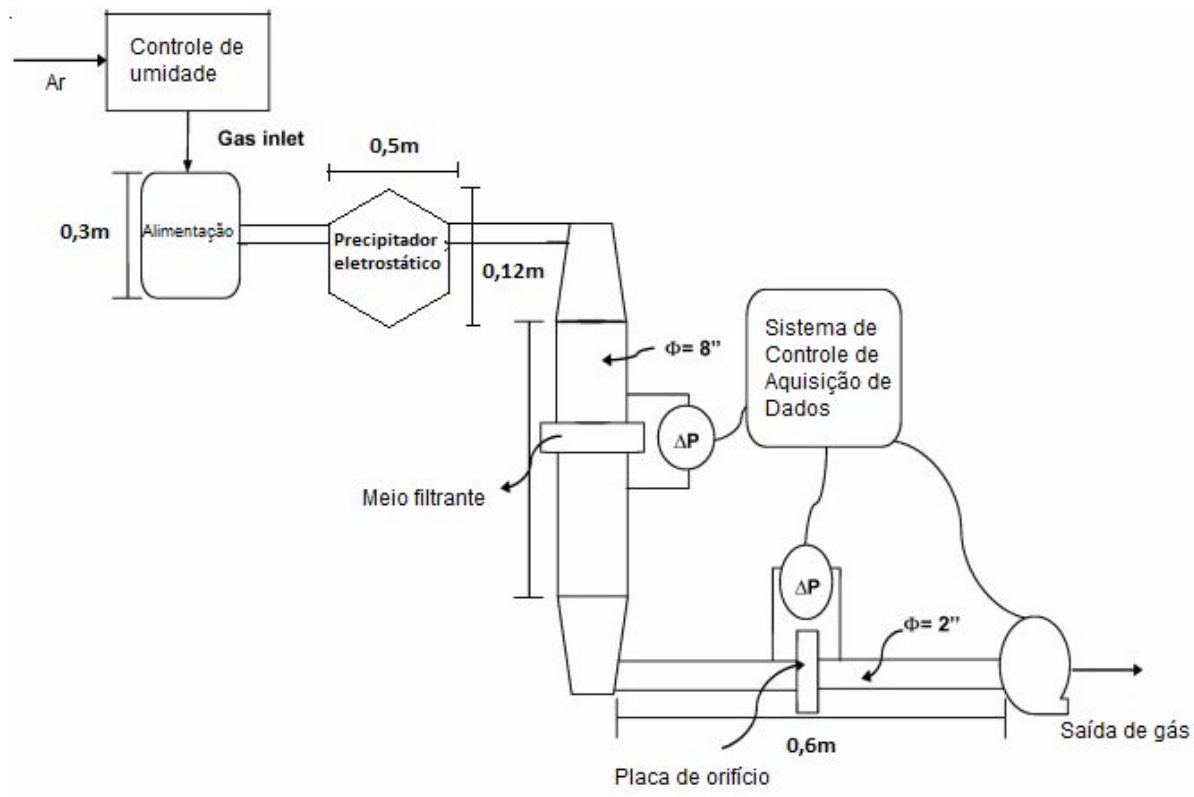

FIGURA 1 - Aparato experimental.

Fonte: Autores.

\section{Fonte de Alta Tensão}

Foi utilizado uma fonte de tensão contínua da marca Spellman, modelo SI1200, para a geração de corona negativa, sua faixa de operação para tensão é de 0 a 50 $\mathrm{kV}$ e para corrente de 0 a $20 \mathrm{~mA}$.

\section{Precipitador Eletrostático}

O equipamento utilizado para o carregamento das partículas do aerossol foi o precipitador eletrostático do tipo placa-fio de estágio único, como demonstrado na Figura 2. 


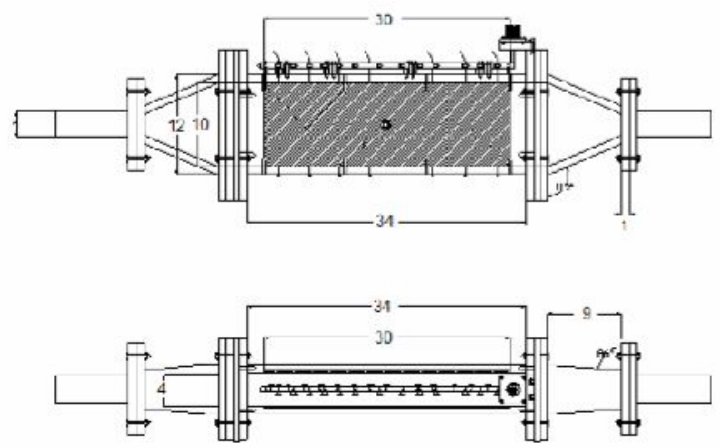

FIGURA 1 - Precipitador eletrostático: esquema de vista lateral (a) e superior com dimensões em (cm) (b) e uma fotografia do precipitador (c).

Fonte: Adaptado de MEIRA, (2009).

A Tabela 1 apresenta as dimensões do precipitador eletrostático: altura, comprimento, distância entre as placas de coleta, diâmetro dos eletrodos de descarga, espaçamento entre os eletrodos de descarga e a distância entre os eletrodos de descarga e a placa de coleta.

TABELA 1 - Dimensões do precipitador eletrostático.

\begin{tabular}{cc}
\hline Dimensão & Medidas $(\mathrm{cm})$ \\
\hline Altura & 30 \\
Comprimento & 10 \\
Distância entre as placas de coleta & 4 \\
Diâmetro dois eletrodos de descarga & 0,025 \\
Espaço entre os eletrodos & 4 \\
Distância entre os eletrodos e a placa coletora & 2 \\
\hline
\end{tabular}

O precipitador eletrostático foi construído em acrílico onde foram colocadas duas placas de cobre nas suas laterais. Estas placas foram conectadas através de fio de cobre revestido a um sistema de aterramento, que consiste de 10 barras de cobre de 2 metros aterradas. Assim as placas funcionavam como eletrodo de coleta. Os eletrodos de descarga, onde era aplicada a tensão consistiam de fios de aço inoxidável colocados no interior da caixa acrílica. Foram utilizados 5 eletrodos de descarga, posicionados verticalmente, paralelos as placas coletoras e igualmente espaçadas entre si com $6 \mathrm{~cm}$.

\section{Material Particulado}

O material particulado utilizado foi o cimento, cedido pela Votorantim Cimentos S.A. de Itaú de Minas (MG) por se tratar de um material comercializado para área de construção civil em todo país. O diâmetro médio volumétrico das partículas de cimento foi obtido pelo equipamento MASTERSIZER MALVERN v.2.19. O diâmetro mediano obtido foi de 14 e $20 \mu \mathrm{m}$. A densidade das partículas foi determinada pelo picnômetro a gás Helio, Accupyc 1330 V3.03, sendo obtido valor de $3100 \mathrm{~kg} / \mathrm{m}^{3}$.

\section{Meio Filtrante}

Os meios filtrantes utilizados nos ensaios de filtração foram filtros de mangas de fibra de vidro, com gramatura de $750 \mathrm{~g} / \mathrm{m}^{2}$. Os meios filtrantes foram cortados em secções circulares de aproximadamente $0,20 \mathrm{~m}$ de diâmetro, com uma área de filtração de $0,0254 \mathrm{~m}^{2}$. 


\section{Métodos}

Foram obtidas curvas de filtração: queda de pressão através do filtro em função da massa retida, sendo o valor máximo de queda de pressão estipulada de $1000 \mathrm{~Pa}$, este valor foi estabelecido pelo trabalho de Oliveira et al., (2015). A partir dos dados obtidos pelas curvas de filtração e pelos parâmetros operacionais, foram calculados a porosidade de Ergun.

Coury (1983) desenvolveu um método que permite estimar a porosidade de tortas de filtração de gases indiretamente, a partir da correlação de Ergun (1952), utilizando a queda de pressão em um leito poroso, como mostra a equação 1:

$$
\frac{\Delta \mathrm{P}}{\mathrm{t}}=150 \cdot \frac{(1-\varepsilon) \mathrm{Q} \mu \mathrm{V}_{\mathrm{F}}}{\mathrm{A} \rho_{\mathrm{P}} \varepsilon^{3} \mathrm{~d}_{\mathrm{P}}^{2}}+1,75 \frac{\mathrm{QP} \mathrm{P}_{\mathrm{g}} \mathrm{V}_{\mathrm{F}}^{2}}{\mathrm{~A} \rho_{\mathrm{P}} \varepsilon^{3} \mathrm{~d}_{\mathrm{P}}}
$$

Sendo que, t é o tempo de filtração, A área de filtração, $Q$ vazão mássica de pó, $P_{g}$ é a densidade do gás, $d_{p}$ é o diâmetro médio das partículas, $\rho_{p}$ densidade da partícula, $\mu$ é a viscosidade do gás; $\varepsilon$ é a porosidade do meio poroso, $V_{F}$ é a velocidade de filtração e $L$ é a espessura da camada porosa.

Também foi calculado através das curvas de filtração a resistência específica da torta (K2), para cada ensaio de filtração. A resistência específica da torta pode ser determinada experimentalmente a partir do aumento da queda de pressão através do meio filtrante devido ao acúmulo de material particulado (SILVA et al., 1999), de acordo com a equação (LEITH et al., 1986):

$$
K_{2 \text { EXPERIMENTAL }}=\frac{A}{V_{F}} * \frac{\Delta P}{\Delta M}
$$

Em que $K_{2}$ é a resistência específica da torta; $A$ é a área de filtração; $V_{F}$ é a velocidade de filtração; $P$ é a queda de pressão do filtro entre dois pontos (início e final da filtração) da parte linear da curva de filtração e $M$ é a variação da massa acumulada por unidade de área em dois pontos.

\section{Testes experimentais}

As partículas foram transportadas por um fluxo de ar seco e carregadas eletrostaticamente pelo carregador eletrostático antes de serem depositadas no filtro para formar a torta de filtração. Os testes foram realizados com velocidade superficial de filtração constante de $0,10 \mathrm{~m} / \mathrm{s}$ e concentração de alimentação de 9-12 $\mathrm{g} / \mathrm{m}^{3}$. Antes de cada experimento, o carregador eletrostático foi limpo com jatos de ar comprimido para evitar qualquer influência de poeira restante da análise anterior.

A umidade relativa do ar na caixa de alimentação foi mantida em aproximadamente $20 \%$, utilizando dois tubos de PVC com sílica gel, que foram acoplados ao equipamento, uma vez que a umidade é um parâmetro importante que influência no carregamento de partículas (WOODHEAD; ARMOUR-CHÉLU, 2003). Antes de cada teste de filtração, a temperatura e a umidade do ambiente e do interior do sistema experimental foram medidas por um termo higrômetro da marca Rotronic, modelo Hygropan, que possui faixas de aplicação entre -10 e $60^{\circ} \mathrm{C}$ e 0 e $100 \%$ U.R. 
O meio filtrante (com massa inicial de aproximadamente $22 \mathrm{~g}$ ) foi colocado na caixa de filtração e os testes foram iniciados após o ajuste da temperatura e umidade. O exaustor foi ligado junto com o alimentador de pó utilizado para dispersar o material particulado na linha de filtração. $O$ ar que continha o material particulado então fluía através do carregador eletrostático localizado entre a caixa de alimentação e a caixa de filtração. $O$ carregamento das partículas se deu devido à a passagem das partículas no precipitador em que os eletrodos foram submetidos a tensões de $0,-1$ e $-2 \mathrm{kV}$. Depois de passar pelo carregador, as partículas eletrizadas foram depositadas na superfície do filtro, formando a torta de filtração. No final da filtração, quando a queda de pressão máxima preestabelecida foi atingida, a operação de filtração foi interrompida e o filtro foi pesado para obter a massa de pó retido.

\section{Determinação da curva corrente-tensão}

Para se obter o valor exato do início da corrente corona (rompimento dielétrico do gás), foi gerada a curva de corrente-tensão, para a velocidade de gás de $10 \mathrm{~cm} / \mathrm{s}$. A umidade relativa do ar foi mantida em aproximadamente $20 \%$, a temperatura ambiente, medida durante todo e experimento, ficou em terno de um valor médio de $26^{\circ} \mathrm{C}$ e o valor médio da pressão ambiente foi de 0,92atm. Inicialmente, foram realizados ensaios sem a presença de partículas $\left(0 \mathrm{~g} / \mathrm{m}^{3}\right)$ durante o escoamento do gás e, posteriormente, adicionou-se na corrente gasosa partículas de cimento, com uma concentração de de $11,8 \mathrm{~g} / \mathrm{m}^{3}$. Assim foi possível comparar a influência das partículas no comportamento elétrico do sistema. Os valores de corrente eram registrados pela fonte de alta tensão, para cada voltagem aplicada nos eletrodos de descarga, a partir de $-8 \mathrm{kV}$, com aumento de $1 \mathrm{kV}$ até alcançar o rompimento dielétrico do gás, que para o escoamento de ar com partículas ocorreu em $13 \mathrm{kV}$. Todos os testes foram realizados em triplicatas.

\section{Curva corrente $\mathrm{x}$ tensão}

\section{RESULTADOS E DISCUSSÕES}

A Figura 3 exibe as curvas corrente-tensão com desvio padrão de 5-8\%.

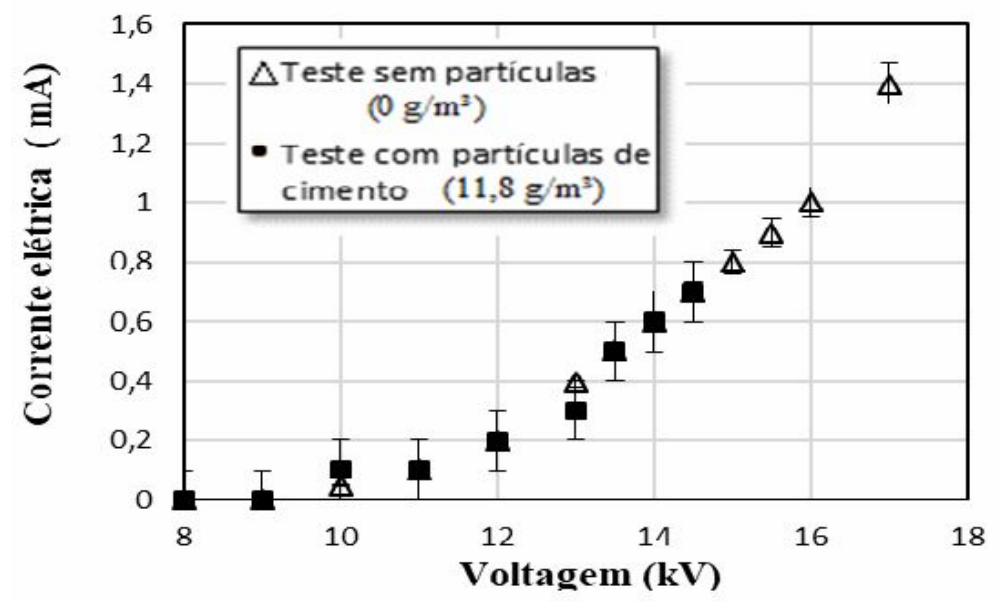

FIGURA 3 - Teste de corrente-tensão para concentração de $0 \mathrm{~g} / \mathrm{m}^{3}$ e 11,8 $\mathrm{g} / \mathrm{m}^{3}$.

O ponto onde se inicia a corrente corona é o ponto exato onde a curva se altera, o valor de corrente vai de 0 para $0,1 \mu \mathrm{A}$, neste teste a corrente corona inicia- 
se com $10 \mathrm{kV}$ aplicados, para ambos os testes, com partículas e sem partículas durante o escoamento de ar. A velocidade do ar foi mantida a $10 \mathrm{~cm} / \mathrm{s}$.

\section{Testes de filtração}

Inicialmente foram realizados ensaios com os filtros sem carga eletrostática (0 $\mathrm{kV}$ ) para os dois tamanhos de partículas. Em seguida, foram utilizadas tensões de $1 \mathrm{kV}$ e $-2 \mathrm{kV}$. É importante ressaltar que embora os valores de tensão utilizados, -1 e -2 kV são inferiores ao valor de início da corrente corona, -10 kV, os resultados apresentados demonstram que pode ocorre eletrização das partículas conforme os trabalhos de Feng et al., (2016), Rodrigues et al., (2017) e Fenara et al., (2011).

A queda de pressão inicial dos filtros limpos foram aproximadamente $148 \mathrm{~Pa}$. Os testes foram realizados em triplicata e os ensaios eram finalizados quando a queda de pressão alcançava $1000 \mathrm{~Pa}$.

As Figuras 4-6 mostram as curvas de filtração para as partículas de 14 e 20 $\mu \mathrm{m}$, sem tensão aplicada $0 \mathrm{kV}$, e tensão aplicada de -1 e $-2 \mathrm{kV}$, respectivamente. Foram realizadas em triplicatas com um desvio padrão de 5\%. Pode observar que ao aplicar a carga eletrostática houve uma mudança no comportamento de formação das tortas.

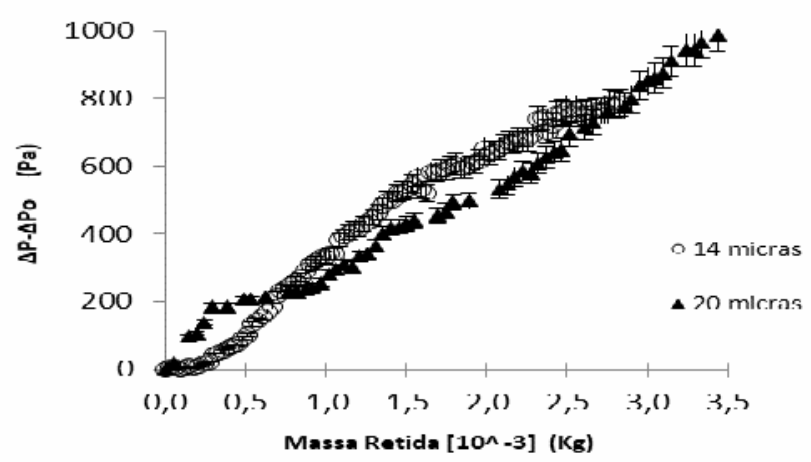

FIGURA 4 - Curvas de filtração com diferentes diâmetros sem aplicação de carga eletrostática $(0 \mathrm{kV})$

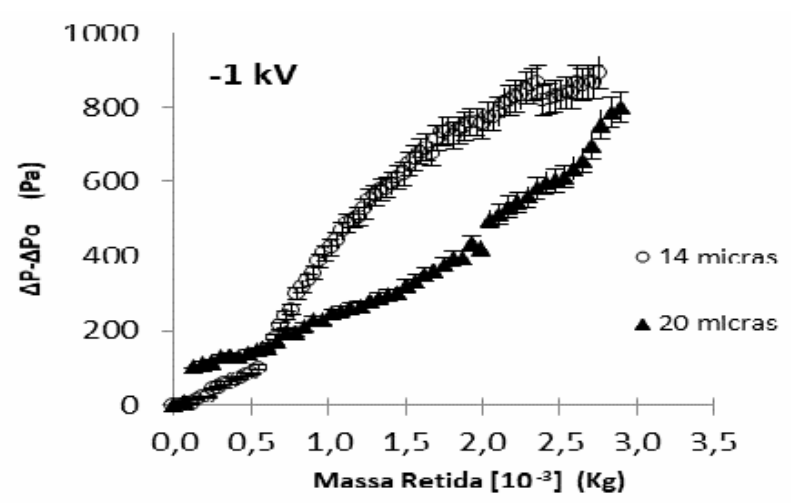

FIGURA 5 - Curvas de filtração com diferentes diâmetros com aplicação de carga de $-1 \mathrm{kV}$. 


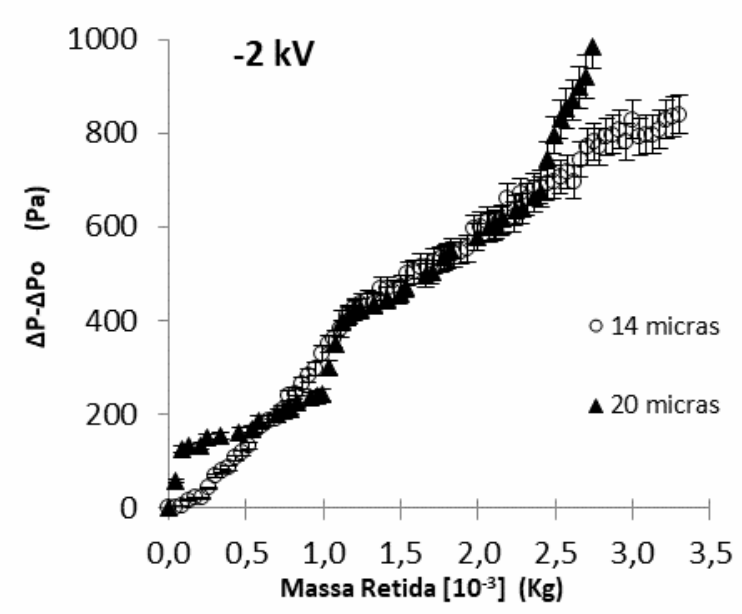

FIGURA 6 - Curvas de filtração com diferentes diâmetros com aplicação de carga eletrostática de $-2 \mathrm{kV}$.

Observando as curvas de filtração das Figs.4-6, pode-se identificar que para os dois tamanhos de partículas houve interferência da carga eletrostática nas curvas de filtração principalmente para $-1 \mathrm{kV}$. Nos estágios iniciais de filtração, todos os testes para partículas de $20 \mu \mathrm{m}$ apresentaram maiores valores de queda de pressão do que para partículas de $14 \mu \mathrm{m}$, para em seguida inverter o comportamento em aproximadamente $200 \mathrm{~Pa}$ de pressão. Este fato pode ser atribuído as maiores partículas obstruírem mais rapidamente os espaços entre as fibras do filtro. Nos testes com aplicação de carga eletrostática de $-2 \mathrm{kV}$ as partículas com $14 \mu \mathrm{m}$ obtiveram menor queda de pressão no final da filtração e maior massa retida, após $700 \mathrm{~Pa}$, quando comparadas as partículas de $20 \mu \mathrm{m}$. Este fenômeno pode ser atribuído ao menor tamanho das partículas de $14 \mu \mathrm{m}$ e a ação das forças eletrostáticas de mesmo sinal. Segundo Mateson et al., (1987) a presença de cargas eletrostáticas nas fibras e/ou nas partículas pode influenciar na formação da torta filtração, alterando a trajetória das partículas, bem como sua ligação partícula-fibra e partícula-partícula. Isso resulta em melhor desempenho em termos de aumento da eficiência do filtro e redução da queda de pressão (SHI; EKBERG, 2015).

$\mathrm{Na}$ Fig. 5 observa-se que com a aplicação de tensão de $-1 \mathrm{kV}$ as partículas de $20 \mu \mathrm{m}$ obtiveram menor queda de pressão durante todo processo de formação da torta, $200<\left(\Delta \mathrm{P}-\Delta \mathrm{P}_{0}\right)<800 \mathrm{~Pa}$. As curvas se distanciaram a partir de uma massa retida de $0,7 \mathrm{~g}$, atingindo a maior diferença em $1,7 \mathrm{~g}$ com 2,8 $\mathrm{g}$ de massa retida atingindo valores próximos de queda de pressão de $850 \mathrm{~Pa}$. Estes resultados concordam com trabalho de Tu et al., (2016; 2018) onde foi observado que para partículas maiores o mecanismo de carregamento das partículas pode ser mais eficiente que para partículas menores.

Para todos os ensaios, a porosidade de Ergun e as resistências específicas das tortas de filtração foram calculadas [Eq.1 e Eq.2] a partir das curvas de filtração obtidas experimentalmente (vide tabela 2). 
TABELA 2- Resultados Experimentais.

\begin{tabular}{|c|c|c|c|c|c|c|}
\hline \multicolumn{7}{|c|}{ Filtro de Fibra de Vidro \# $750 \mathrm{~g} / \mathrm{m}^{3}$} \\
\hline Diâmetro & \multicolumn{3}{|c|}{$14 \mu \mathrm{m}$} & \multicolumn{3}{|c|}{$20 \mu \mathrm{m}$} \\
\hline $\begin{array}{c}\text { Tensão } \\
(\mathrm{kV})\end{array}$ & 0 & -1 & -2 & 0 & -1 & -2 \\
\hline $\mathrm{K} 2\left(10^{3} \mathrm{~s}^{-3}\right)$ & 70,5 & 82,3 & 64,5 & 73,3 & 70,4 & 91,3 \\
\hline$\varepsilon$ Ergun & 0,46 & 0,45 & 0,48 & 0,50 & 0,51 & 0,48 \\
\hline $\begin{array}{c}\text { Massa } \\
\text { Retida (g) }\end{array}$ & 2,82 & 2,80 & 3,30 & 3,40 & 2,90 & 2,70 \\
\hline
\end{tabular}

Com a aplicação de tensão de -2 kV os resultados foram contrários aos obtidos com tensão de $-1 \mathrm{kV}$. Pode ser visto na Tab. 2 que a resistência específica e a porosidade das tortas obtiveram um comportamento diferente para cada tamanho de partícula. Para partículas de $14 \mu \mathrm{m}$ a resistência específica aumentou $17 \%$ e, em seguida, reduziu $28 \%$, com o aumento da tensão aplicada. No caso de partículas de $20 \mu \mathrm{m}$, a resistência específica reduziu $5 \%$ de 0 para $-1 \mathrm{kV}$ e aumentou $30 \%$ quando a tensão foi de $-2 \mathrm{kV}$.

A resistência específica para partículas com $14 \mu \mathrm{m}$ de diâmetro diminuiu em $9 \%$ comparando a curva sem carga eletrostática com a curva de $-2 \mathrm{kV}$ (vide Fig. 7), porém para curva de $-1 \mathrm{kV}$ a resistência aumentou cerca de $16 \%$, o aumento da porosidade e a diminuição da resistência específica, faz com que o filtro retenha mais partículas durante a filtração, melhorando sua eficiência de filtração.

Com a aplicação de $-1 \mathrm{kV}$ os resultados de queda de pressão foram contrários aos obtidos para tensão de $-2 \mathrm{kV}$ em relação ao teste sem aplicação de tensão $(0$ $\mathrm{kV}$ ). A queda de pressão para partículas de $20 \mu \mathrm{m}$ diminuiu em $4 \%$ comparando a curva sem carga eletrostática com a curva de $-2 \mathrm{kV}$ (vide Fig. 8), porém para curva de $-2 \mathrm{kV}$ a resistência aumentou cerca de $24 \%$, o aumento da porosidade e a diminuição da resistência específica, faz com que o filtro retenha mais partículas durante a filtração, melhorando sua eficiência de filtração.

Os valores de Ergun comprovaram que a torta ficou menos compactada, facilitando o escoamento e a passagem do ar, à medida que aumentou a carga eletrostática, aumentou a porosidade de Ergun e diminuiu a resistência específica da torta, em alguns casos. Porém isto não foi observado para todos os tamanhos de partículas, para partículas de $20 \mu \mathrm{m}$ isto ocorreu somente na curva de $-1 \mathrm{kV}$, e para partículas de $14 \mu \mathrm{m}$ foi visto na curva de $-2 \mathrm{kV}$.

Como apresentado na Tabela 2, para o filtro de fibra de vidro a menor resistência específica e maior massa retida foram obtidas para partícula de $14 \mu \mathrm{m}$ com uma carga de $-2 \mathrm{kV}$, obtendo uma resistência específica menor em $9 \%$ comparando com a curva sem carga eletrostática aplicada e uma massa retida de $17 \%$ a mais que a curva sem carga.

Esta mudança de comportamento existe devido às diferenças de tamanho de partícula, e pode ser vista no trabalho de Feng et.al. (2016), onde foi utilizado uma variação do tamanho de partículas entre 0 e $3 \mu \mathrm{m}$ e uma carga eletrostática de -6,5 $\mathrm{kV}$, os autores observaram uma mudança no comportamento de formação das tortas em relação ao tamanho das partículas e na eficiência de filtração, com partículas maiores Feng el al. (2016) obtiveram eficiências de filtração melhores chegando a $80 \%$ de eficiência, quando comparados aos ensaios sem carga eletrostática aplicada. Nas Figuras 7 e 8 são apresentados os gráficos separadamente para cada tamanho de partículas com as alterações de cargas aplicadas, respectivamente. 


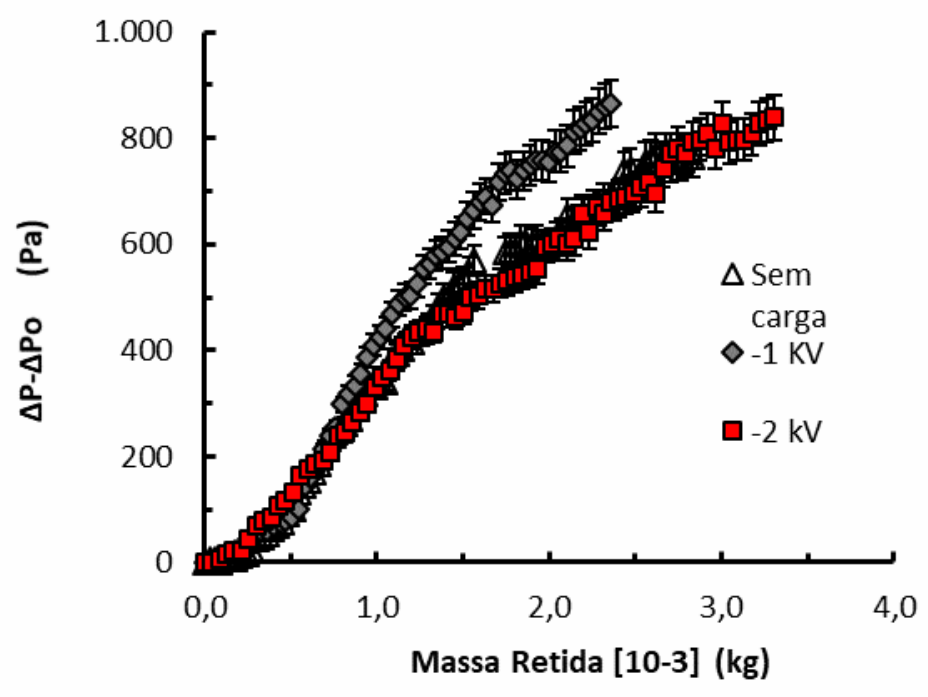

FIGURA 7 - Curvas de filtração para diâmetro médio de partículas de cimento de 14 $\mu \mathrm{m}$.

$\mathrm{Na}$ Figura 7, pode ser observado que para as partículas de $14 \mu \mathrm{m}$, as curvas de 0 e $-2 \mathrm{kV}$ não obtiveram alterações em relação à carga aplicada. Acredita-se que este comportamento esteja relacionado com o tamanho das partículas e com a velocidade de filtração.

Estudos anteriores (GIVEHCHI et al., 2015; YANG et al., 2018) demonstraram a influência do tempo de residência das partículas em precipitadores eletrostáticos, o tempo em que a partícula fica no interior do filtro é fundamental para a obtenção dos resultados mais eficientes.

Neste caso, com uma carga menor de $-1 \mathrm{kV}$ conseguiu-se aumentar a queda de pressão, podendo-se dizer que as partículas foram carregadas para esta tensão e obtiveram uma maior queda de pressão devido a sua formação da torta, visto que, com a carga de $-1 \mathrm{kV}$ foi obtido a menor porosidade da torta de 0,45 , isto faz com que a resistência do ar aumente, aumentando a queda de pressão durante a filtração de gases.

Para partículas com diâmetro maior $(20 \mu \mathrm{m})$ como apresentado na Figura 8, o resultado obtido foi o inverso, a curva com maior queda de pressão foi a de $-2 \mathrm{kV}$, que obteve um aumento de $24,5 \%$ na sua resistência específica da torta (k2) e diminuiu $4 \%$ em sua porosidade, em relação à curva sem carga eletrostática aplicada. 


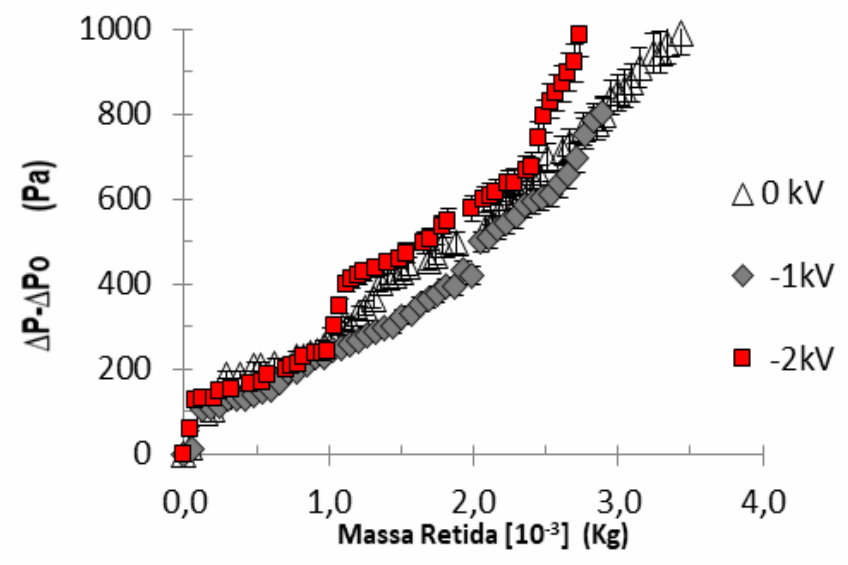

FIGURA 8 - Curvas de filtração para diâmetro médio de partículas de cimento de 20 $\mu \mathrm{m}$.

A curva de $-1 \mathrm{kV}$ obteve a menor resistência específica neste caso, diminuindo em $4 \%$ comparando com a curva sem carga. Henry e Ariman (1986) em seus estudos, afirmam que a ação de um campo elétrico na filtração de ar em tecidos, tende a diminuir a densidade de empacotamento das partículas, proporcionando assim uma menor resistência ao escoamento do ar.

Este resultado também foi obtido por outros pesquisadores (RODRIGUES et al., 2017), com mesmo comportamento, ou seja, a formação da torta foi mais favorável com a condição experimental sem carregamento eletrostático nas partículas pois quando os autores aplicaram uma carga de - -3 e $-6 \mathrm{kV}$ não obtiveram melhorias nos resultados.

\section{CONCLUSÕES}

O trabalho apresentou resultados positivos na aplicação de cargas eletrostáticas nas partículas, comprovando que o tamanho das partículas e a carga eletrostática afetam diretamente na formação da torta, porém com uma faixa de tamanho de partículas estabelecido, pode-se obter uma diminuição na queda de pressão e um aumento na massa retida, para o filtro de fibra de vidro.

Com base nestes estudos, para um diâmetro volumétrico de $14 \mu \mathrm{m}$ a melhor voltagem para se trabalhar foi de $-2 \mathrm{kV}$, atingindo os objetivos do trabalho na diminuição da resistência específica da torta e no aumento da massa retida. Toda via, para o diâmetro volumétrico de $20 \mu \mathrm{m}$ a melhor condição de trabalho foi a condição experimental de $-1 \mathrm{kV}$ aplicado, visto que conseguiu-se aumentar a porosidade da torta e diminuir a resistência específica da torta.

O trabalho possibilitou a otimização da filtração com a aplicação da carga eletrostática nas partículas de cimento, visto que este processo ainda não foi estudado para o mesmo material particulado, foi possível alcançar uma melhoria na deposição das partículas no filtro formando tortas mais porosas e com uma menor resistência específica ao fluxo de ar, podendo facilitar a limpeza das mangas, aumentando a vida útil das mesmas e diminuindo o gasto energético no processo. 


\section{AGRADECIMENTOS}

Os autores agradecem o Departamento de Engenharia Química da Universidade Federal de São Carlos, bem como as agências de fomento e incentivo a pesquisa, CNPq e CAPES.

\section{REFERÊNCIAS}

AGUIAR M. L.; COURY J. R. Cake formation in fabric filtration of gases. Industrial Chemical Engineering Research 35, 3673-3679, 1996.

BARROS P; TANABE E; AGUIAR M Performance of fibrous filters during nanoparticle cake formation. Separation Science and Technology (Philadelphia) (2016). DOI: 10.1080/01496395.2015.1119845

BROWN, R.C.; GRAY, W. R.; BLACKFORD, D. B.; BOSTOCK, G. J. Effect of industrial aerosols on the performance of electrically charged filter material. Annals of Occupational Hygiene 32, 271-294, 1988.

COURY. J. R. Electrostatic effect in granular bed filtration of gases. Tese de Doutorado. Cambridge university. 1983.

FENARA, B. C, RODRIGUES, M.V, AGUIAR, M.L., Study of the electrostatic e Effect in the filtration of micrometer particles. Proceedings, FILTECH, March 2224 2011, Wiesbaden, Germany.

FENG, Z; LONGA, Z; YUB, T. Filtration characteristics of fibrous filter following an electrostatic precipitator. Journal of Electrostatic 83 52-62, 2016. DOI: 10.1016/j.elstat.2016.07.009

FENG, Z.; PAN, W.; ZHANG, H.; CHENG, X.; LONG, Z.; MO. J. Evaluation of the performance of an electrostatic enhanced air filter (EEAF) by a numerical method. Powder Technology 327 (2018) 201-214. DOI: 10.1016/j.powtec.2017.12.054

FREDERICK, E. R. Some Effects of Electrostatic Charges In Fabric Filtration, Journal of the Air Pollution Control Association, 24:12, 1164-1168, (1974) DOI: 10.1080/00022470.1974.10470030

GIVEHCHI, R. LI, Q., TAN, Z. The effect of electrostatic forces on filtration efficiency of granular filters. Powder Technology (2015). Vol 277, June 01, pg 135-140. DOI: 10.1016/j.powtec.2015.01.074J. U

INNOCENTINI M. D M.; TANABE E.; AGUIAR M. L.; COURY J. R. Filtration of gases at high pressures: Permeation behavior of fiber-based media used for natural gas cleaning. Chemical Engineering Science, vol. 74, 38-48 2012. DOI: 10.1016/j.ces.2012.01.050

JAWOREK, A.; KRUPA, A.; CZECH, T. A.T. SOBCZYKA, A. MARCHEWICZA, L. ŚLIWIŃSKI. Hybrid electrostatic filtration systems for fly ash particles emission control. A review, Separation and Purification Technology, 213 283-302, 2019. DOI: 10.1016/j.seppur.2018.12.011 
JAWOREK, A.; MARCHEWICZ, A.; SOBCZYK, A. T.; KRUPA, A.T.; CZECH T. Twostage electrostatic precipitator with dual-corona particle precharger for PM2.5 particles removal Journal of Cleaner Prodution. 164 (2017), p. 1645-1664, DOI: 10.1016/j.jclepro.2017.07.032

LEITH, D.; ALLEN, R. W. K. Dust filtration by Fabric Filters. Progress in Filtration and Separation, v.4, p.1-55,1986.

MATESON, M. J OR. C. Filtration: Principles and Practices. 2nd ed. New York: Dekker, 1987.

MEIRA, C. R. de. Desempenho de um precipitador eletrostático operando na remoção de nanopartículas de aerossóis. 106 p. Tese (Mestrado em Engenharia Química). UFSCar, São Carlos, 2009.

OLIVEIRA, F. M., CIRQUEIRA, S. S. R., AGUIAR, M. L. Effect of electrostatic charging in filtration of cement particles using bag filters. Proceedings, FILTECH, February 24-26 (2015), Cologne, Germany.

RODRIGUES M.V.; BARROZO M.A.S.; GONÇALVES J.A.S.; COURY J.R. Effect of particle electrostatic charge on aerosol filtration by a fibrous filter. Powder Technology 313, 323-331, 2017. DOI: 10.1016/j.powtec.2017.03.033

SCHMIDT, E. LÖFFLER, F. Preparation of dust cakes for microscopic examination, Powder Technology 60, 173-177, (1990). Doi: 10.1016/0032- 5910(90)80141-K

SHI, B. EKBERG, L. Ionizer assisted air filtration for filtration of submicron and ultrafine particles-evaluation of long-term performance and influencing factors, Environmental Science Technology. $49 \quad$ (11) (2015) 6891-6898. DOI: $10.1021 /$ acs.est.5b00974

SILVA, C. R. N.; NEGRINI, V. S.; AGUIAR, M. L.; COURY, J. R. Influence of gas velocity on cake formation and detachment. Powder Technology, v.101, p. 165-172, 1999. DOI: 10.1016/S0032-5910(98)00168-5

TU, G.; SONG, Q.; YAO, Q. Mechanism study of electrostatic precipitation in a compact hybrid particulate collector. Original Research Article Powder Technology, Volume 328, P.84-94, 1 April 2018. DOI: 10.1016/j.powtec.2018.01.016

TU, G.; SONG, Q.; YAO, Q. Relationship between particle charge and electrostatic enhancement of filter performance. Powder technology 301, 665-673, 2016. DOI: 10.1016/j.powtec.2016.06.044

WOODHEAD, S. R.; ARMOUR-CHÉLU, D. I. The influence of humidity, temperature and other variables on the electric charging characteristics of particulate aluminium hydroxide in gas-solid pipeline flows. Journal of Electrostatics, 58, p. 171-183, 2003. DOI: 10.1016/S0304-3886(03)00046-9 
YANG. Z.; ZHENG, C.; ZHANG, X.; ZHOU, H.; SILVA, A. A.; LIU, C.; SNYDER, B.; WANG, Y.; GAO, X. Challenge of $\mathrm{SO} 3$ removal by wet electrostatic precipitator under simulated flue gas with high SO3 concentration. Fuel, v. 217, p. $597-604,2018$. DOI: 10.1016/j.fuel.2017.12.125 